Check for updates

Cite this: RSC Adv., 2017, 7, 35704

Received 20th April 2017 Accepted 12th July 2017

DOI: $10.1039 / \mathrm{c} 7 \mathrm{ra0} 4444 \mathrm{e}$

rsc.li/rsc-advances

\section{A redox responsive controlled release system using mesoporous silica nanoparticles capped with Au nanoparticles}

\begin{abstract}
Hongnan Qu, (D) $\dagger^{\text {abcd }}$ Liangrong Yang, $\dagger^{\mathrm{b}}$ Jiemiao Yu, ${ }^{\mathrm{b}}$ Tingting Dong, ${ }^{\mathrm{bc}}$ Meng Rong, ${ }^{\mathrm{bc}}$ Jianfeng Zhang, ${ }^{\text {bcd }}$ Huifang Xing, ${ }^{\mathrm{b}}$ Li Wang, ${ }^{\mathrm{b}}$ Feng Pan $^{\mathrm{b}}$ and Huizhou Liu*b

A novel redox responsive controlled release system based on the host-guest interaction between ferrocene modified mesoporous silica nanoparticles (MSN-FC) and $\beta$-cyclodextrin modified gold nanoparticles (Au@CD) was developed. FITC was used as the release molecule and it was loaded into the pores of MSN-Fc. With the addition of oxidant $\left(\mathrm{H}_{2} \mathrm{O}_{2}\right)$, oxidized ferrocene leaves the hydrophobic cavity of $\beta-C D$, resulting in cargo controlled release. Due to the structural properties of the modified MSN, the continuously slow release behavior showed the prospect to be a sustained drug delivery system. This novel redox responsive system possessed stimuli-responsive controlled release and sustained release properties, and therefore has potential applications in sustained and targeted drug delivery.
\end{abstract}

\section{Introduction}

Since the first report using MCM-41 type mesoporous silica nanoparticles (MSN, size typically $<500 \mathrm{~nm}$ ) as a drug system in $2001,{ }^{1}$ MSN has attracted substantial attention in recent years due to their advantageous structural properties, such as large surface areas and pore volume, tunable pore sizes, colloidal stability, good biocompatibility and the possibility to specifically functionalize the inner pore system and the external particle surface. These highly attractive features make MSN a promising and widely applicable platform for drug delivery. ${ }^{2}$ Due to the simple post-modification of the surface, MSN has been designed as a smart responsive control release system in recent years. ${ }^{3}$ The changes in interactions within the responsive system can be triggered by applying an appropriate stimuluses such as temperature, $\mathrm{pH}$, light, magnetic field, enzyme and redox. ${ }^{4-11}$ As one type of novel stimulus, redox can drive the structural changes of the system through inducing electron transfer reactions. The past researches showed that the strongly redox potential gradient between tumor cells and normal cells due to the overproduction of intracellular glutathione (GSH), dithiothreitol (DTT) or reactive oxygen species (ROS) has been extensively explored for designing redox responsive controlled

\footnotetext{
${ }^{a}$ Qingdao Institute of Bioenergy and Bioprocess Technology, Chinese Academy of Sciences, Qingdao 266101, China

${ }^{b}$ Key Laboratory of Green Process and Engineering, State Key Laboratory of Biochemical Engineering, Institute of Process Engineering, Chinese Academy of Sciences, Beijing 100190, China. E-mail: hzliu@ipe.ac.cn

'University of Chinese Academy of Sciences, Beijing, 100049, China

${ }^{d}$ Sino-Danish Center for Education and Research, Beijing, 100190, China

$\dagger$ These authors are contributed equally to this study.
}

release system. ${ }^{12,13}$ The past couple years have witnessed a tremendous process in the development of GSH/DTT responsive drug delivery based on disulfide bond cleavage. For example, mesoporous silica nanorods capped with superparamagnetic iron oxide nanoparticles can act as a redoxresponsive controlled release delivery carrier using cellproduced antioxidants DTT as triggers. ${ }^{14}$ The capping system based on MSN has proved an excellent method for blocking the drugs in the pore channel. Inorganic nanoparticles such as gold nanoparticles (AuNPs) ${ }^{15,16}$ which present fascinating aspects such as size-related electronic, magnetic and optical properties can be chemically attached on the MSN surface as gatekeepers to block the pore entrances for efficient sealing of the interior mesoporous environment. Reports also showed that the redox responsive valve using polymer network which contained disulfide bonds can be cleaved by DTT. Feng and coworkers reported redox-responsive nanogated MSN by grafting poly $(N-$ acryloxysuccinimide) to the pore entrance of MSN followed by crosslinking with cystamine and the loaded rhodamine B was rapidly released in response to $21.6 \mathrm{mM}$ DTT. ${ }^{17}$ Cui and collaborators reported a novel PEG surface-capped mesoporous silica based nanosystem, where the PEG cappers were linked to the MSN by disulfide bonds which were inclined to be cleaved under GSH environment. ${ }^{18}$ Another report revealed a redox responsive system based on SBA-15 with cysteine ester linked mPEG chains can also be triggered by GSH. ${ }^{19}$ However, these drug delivery systems based on polymers usually face the challenges of aggregation, rapid clearance during circulation and burst release.

Very recently, supramolecular constructed by host-guest interactions is also an important way to construct stimuliresponsive systems. ${ }^{20,21}$ In this regard, materials grafted on 
the surfaces of MSN include host molecules such as pillararenes (PA), ${ }^{22,23}$ crown ethers, ${ }^{24}$ cucurbiturils (CB), ${ }^{25,26}$ calixarenes (CA), ${ }^{27}$ cyclodextrins $(\mathrm{CD})^{28}$ or a range of stimuli-responsive guest molecules. In particular, several reports showed that the properties of ferrocene (Fc) containing redox molecular systems can be manipulated by altering the oxidation state of redoxactive ferrocene moieties. Fc was widely investigated in redox responsive system as an important guest molecule which can interact with different host components. ${ }^{29-32}$ Among which CDs are commercially available, and the hydroxyl groups offer them the opportunity to be easily modified. Particularly, $\beta$-CD has the strongest binding ability under $1: 1$ molar ratio of $\beta$-CD and Fc. $^{33}$ Normally, uncharged Fc species or its derivatives can strongly bound into the cavity of $\beta-\mathrm{CD}$, whereas the charged species $\left(\mathrm{Fc}^{+}\right)$will rapidly dissociate out of the cavity, and this process can be reversibly switched chemically or electrochemically. ${ }^{30,34}$ Yang has developed a smart $\mathrm{pH}$ and sugar responsive cargo release hybrid nanomaterial which used $\mathrm{Fe}_{3} \mathrm{O}_{4}$ nanoparticles as core and MSN as the shell followed by the covalent installation of a layer of $\beta$-CD on the outer surfaces. When the $\mathrm{pH}$ is lower than 8.0, the cargo is immediately released in 2 hours. ${ }^{35}$ Yan constructed a voltage responsive system based on the host-guest interaction between $\beta$-CD and Fc, which can complete released of rhodamine $\mathrm{B}$ from $450 \mathrm{~min}$ to $32 \mathrm{~min}$ when the voltage increased from $+1.0 \mathrm{~V}$ to $+4.0 \mathrm{~V}^{20}$ Zhuo et al. reported a dual-stimulated release system of size-selected cargos from $\beta$-CD covered MSN. The cavity of $\beta$-CD was blocked by Fc and the cargo can be quickly released by the escape of Fc under electro-stimuli or the detachment of $\beta$-CD with UV irradiation. ${ }^{36}$ Studies in drug controlled release system based on the host-gust interaction were inclined to have a short release period. However, these drug nanocarriers are required to be smart, which not only can response to intracellular environmental stimuli to realize target release but also can achieve a prolonged therapeutic effect by slow release of the therapeutic substance over an extended duration after administration of a single dose to achieve lesser frequency of administration.

Herein, we present a novel controlled and sustained drug delivery system based on host-guest interaction between Fc and $\beta$-CD. MSN which act as a container for drug molecules were modified with ferrocene derivative groups. $\beta$-CD functionalized AuNP was used as the gatekeeper to block the pores of the MSN. AuNP modified with $\beta$-CD would be capped on the pores of MSN when the ferrocene molecules formed the stable interaction complex with $\beta$-CD under redox environment. On the contrary, when the ferrocene oxidized to the hydrophilic $\mathrm{Fc}^{+}$group with addition of $\mathrm{H}_{2} \mathrm{O}_{2}$, the $\mathrm{Fc}^{+}$group will leave the hydrophobic cavity of $\beta-\mathrm{CD}$, resulting in the cargo releasing.

\section{Materials and methods}

\section{Chemicals and reagents}

The cetyltrimethylammonium bromide (CTAB), fluorescein isothiocyanate isomer I (FITC), 3-aminopropyltriethoxysilane (APTES) were purchased from Sigma-Aldrich. The thiol- $\beta$ cyclodextrin (SH- $\beta-\mathrm{CD})$ was obtained from Shandong Binzhou Zhiyuan Bio-Technology Limited (China). The chloroauric acid
( $\mathrm{HAuCl}_{4} \cdot 4 \mathrm{H}_{2} \mathrm{O}$ ), tetraethylorthosilicate (TEOS), sodium citrate, hydrogen peroxide (30\%), ferrocenecarboxaldehyde (Fc-CHO) and other analytical-grade solvents were from Sinopharm chemical reagent Co., Ltd. All of the chemicals were used without further purification.

\section{Synthesis of amino-functionalized mesoporous silica nanoparticles (MSN-NH ${ }_{2}$ )}

The mesoporous silica nanoparticles (MSN) were synthesized by using $\mathrm{CTAB}$ as a template according to previous reports. ${ }^{37}$ Specifically, $1.00 \mathrm{~g}$ of CTAB was dissolved in $480 \mathrm{ml}$ of deionized water. Then $3.5 \mathrm{ml} 2 \mathrm{M} \mathrm{NaOH}$ was added into the CTAB solution and the mixture was heated to $80{ }^{\circ} \mathrm{C}$ while stirring for $30 \mathrm{~min}$. Then, $5 \mathrm{ml}$ of TEOS was added dropwise into the aqueous solution. The mixture solution gradually turned white and kept at $80{ }^{\circ} \mathrm{C}$ for another $2 \mathrm{~h}$. The products were filtered and centrifuged, and washed with ethanol and deionized water for several times. Finally, the product was dried under high vacuum at $60{ }^{\circ} \mathrm{C}$ for $12 \mathrm{~h}$. To remove template CTAB, the white solid was heated at $550{ }^{\circ} \mathrm{C}$ for $5 \mathrm{~h}$. Next, the $\mathrm{MSN}-\mathrm{NH}_{2}$ was prepared according to a reported literature procedures with a slight modification..$^{38,39} 0.5 \mathrm{~g}$ MSN was solved into $50 \mathrm{ml}$ acetonitrile, and the excess of 3-aminopropyltriethoxysilane (APTES) was added to the solution. The mixture was stirred for $24 \mathrm{~h}$ to yield MSN-NH$H_{2}$. The resulting particles were separated by centrifugation, washed with deionized water and ethanol, and dried under high vacuum at $60{ }^{\circ} \mathrm{C}$.

\section{Synthesis of ferrocenecarboxaldehyde modified MSN} (MSN-Fc)

$150 \mathrm{mg}$ MSN-NH $\mathrm{H}_{2}$ and $400 \mathrm{mg}$ ferrocenecarboxaldehyde were dissolved in $10 \mathrm{ml}$ ethanol and the mixture was stirred at $60{ }^{\circ} \mathrm{C}$ for $6 \mathrm{~h}$. The mixture was then washed with ethanol and isopropyl alcohol (IPA), and immersed in a solution of sodium borohydride $(0.1 \mathrm{mM})$ in methanol for $1 \mathrm{~h}$. Subsequently, the newly obtained MSN-Fc was washed with methanol, water and IPA several times, and dried under high vacuum at $60{ }^{\circ} \mathrm{C}$ for $24 \mathrm{~h}$.

\section{Preparation of $\beta$-cyclodextrin modified gold nanoparticles (AuNP@CD)}

AuNP was prepared by citrate reduction of $\mathrm{HAuCl}_{4}$ according to a reported protocol with slight modifications. ${ }^{40}$ All glassware used in these preparations was cleaned in aqua regia (3 parts $\mathrm{HCl}, 1$ part $\mathrm{HNO}_{3}$ ), rinsed in triply Millipore-filtered water, and oven-dried prior to use. In a $500 \mathrm{ml}$ round-bottom flask equipped with a condenser, $250 \mathrm{ml}$ of $0.01 \% \mathrm{HAuCl}_{4}$ was brought to a boil with vigorous stirring. $4.5 \mathrm{ml}$ of $1 \%$ sodium citrate was added to this solution. The solution turned blue within $20 \mathrm{~s}$ and turned wine red after $60 \mathrm{~s}$. After boiling for an additional $10 \mathrm{~min}$, the heating source was removed, and the solution was stirred for another $15 \mathrm{~min}$. Next, the AuNP@CD was prepared following the literature. ${ }^{\mathbf{4 0}} 10 \mathrm{mg} \mathrm{SH}-\beta-\mathrm{CD}$ was added to the $100 \mathrm{ml} 10 \mathrm{nM}$ AuNP solutions, and the mixture was stirred for $24 \mathrm{~h}$ at $50{ }^{\circ} \mathrm{C}$. Then, the resulting mixture was collected by centrifugation, and the precipitate was washed with $0.01 \%$ SDS 
solution for three times. The AuNP@CD was dissolved in deionized water and stored at $4{ }^{\circ} \mathrm{C}$.

\section{FITC loading and AuNP@CD capping}

$10 \mathrm{mg}$ MSN-Fc and $1 \mathrm{mg}$ FITC were dissolved in PBS buffer ( $\mathrm{pH}$ $=7.4$ ), followed by the sonication of the mixture for $30 \mathrm{~min}$. The resulting solution was stirred for $24 \mathrm{~h}$ at room temperature. Next, in order to remove the free FITC, the MSN-Fc was collected using centrifugation at $5000 \mathrm{rpm}$ for $5 \mathrm{~min}$, and washed with deionized water and PBS buffer for several times. The loaded MSN-Fc was dried under high vacuum at $60{ }^{\circ} \mathrm{C}$ for $12 \mathrm{~h}$. Afterwards, $5 \mathrm{mg}$ loaded MSN-Fc was added into $10 \mathrm{ml} 10 \mathrm{nM}$ AuNP@CD solution, and stirred for $12 \mathrm{~h}$. In order to cover completely, the mixture was collected by centrifugation and another fresh AuNP@CD solution was added. Finally, the resulting AuNP@CD capped FITC-loaded MSN-Fc was centrifuged at $5000 \mathrm{rpm}$ for $5 \mathrm{~min}$, and washed with deionized water at least three times.

\section{Controlled release experiment}

$1 \mathrm{mg}$ of FITC-load and AuNP@CD-capped MSN-Fc was dispersed in $10 \mathrm{ml}$ of PBS buffer ( $\mathrm{pH}=7.4$ ) and added $3 \mu \mathrm{l}$ of $\mathrm{H}_{2} \mathrm{O}_{2}$ at room temperature. Subsequently, $300 \mu$ l of solution was taken periodically every $2 \mathrm{~h}$ from the suspension followed by centrifugation (12 $000 \mathrm{rpm}, 5 \mathrm{~min}$ ). The release of FITC from the pores to the buffer solution was determined by fluorescence emission spectroscopy (ex at $490 \mathrm{~nm}$ ).

\section{Characterization}

The UV-visible spectrum was obtained from HITACHI U-4100 (Japan). The transmission electron microscopy (TEM) images were obtained on a JEM-2100F electron microscope (Japan) operating at an accelerating voltage of $200 \mathrm{kV}$. X-ray photoelectron spectroscopy (XPS) spectra were recorded on an ESCALAB 250Xi (Thermo Fisher) using a monochromatic Al$\mathrm{K} \alpha(1486.8 \mathrm{eV}) \mathrm{X}$-ray source at power of $225 \mathrm{~W}$. The C1s line at $284.6 \mathrm{eV}$ was used as the binding energy reference and C1s, O1s, N1s, Si2p, Fe2p and Au4f regions were acquired. Nitrogen adsorption-desorption isotherms were carried out on an automatic surface and aperture distribution analyzer (Autosorb-IQ-MP) at $77 \mathrm{~K}$ under a continuous adsorption condition. Prior to measurement, all samples were degassed for $24 \mathrm{~h}$ in a vacuum oven before measurement. The pore size distribution was calculated from adsorption branches of isotherms by the Barrett-Joyner-Halenda (BJH) method. Pore volume and specific surface area were calculated with Brunauer-Emmett-Teller and Barrett-Joyner-Halenda (BETBJH) methods. The FITR spectra were obtained from BRUKER TENSOR 27 and fluorescence data was obtained from HITACHI F-7000 (Japan). The zeta potential was measured using the DelsaNano C (Beckman Coulter, Inc.). Before zeta potential measurement all samples were sonicated for 10 minutes.

\section{Results and discussion}

\section{Synthesis, characterization and functionalization of MSN}

In this study, we synthesized a controlled release system based on the host-guest interaction between Fc modified on MSN (MSN-Fc) and $\beta$-cyclodextrin modified on the gold nanoparticles (AuNP@CD). We synthesized $\mathrm{MSN}-\mathrm{NH}_{2}$ by modifying MSN using APTES and obtained the host material MSN-Fc through grafting $\mathrm{Fc}$ on the MSN-NH . The AuNP was synthesized and functionalized with thiol group modified $\beta$-cyclodextrin (AuNP@CD), which can interact with Fc of MSN-Fc when it is under redox state through host-guest interaction. When the ferrocene oxidized to the hydrophilic $\mathrm{Fc}^{+}$group with addition of $\mathrm{H}_{2} \mathrm{O}_{2}$, the $\mathrm{Fc}^{+}$group will leave the hydrophobic cavity of $\beta-\mathrm{CD}$, resulting in cargo releasing (Scheme 1).

For the preparation of $13 \mathrm{~nm}$ AuNP, we choose the most popular method according to the previous reports which reduce the $\mathrm{HAuCl}_{4}$ by citrate under boiling conditions. Au@CD was obtained by functionalized with thiol group modified $\beta$-cyclodextrin. As shown in Fig. 1, the UV-visible spectrum indicated that the AuNP exhibited a maximum peak of the surface plasmon resonance absorption at $518 \mathrm{~nm}$ and it was red-shifted to $522 \mathrm{~nm}$ for Au@CD. This result was consistent with the previous reported data ${ }^{\mathbf{4 0}}$ and demonstrated that AuNP was synthesized successfully. Meanwhile, the average dynamic light scattering (DLS) size before and after modification of $\beta$-cyclodextrin was about $27 \mathrm{~nm}$ and $34 \mathrm{~nm}$, respectively. Huang et al. reported when the UV-vis spectrum of AuNP blue-shifted $10 \mathrm{~nm}$ (528-518 $\mathrm{nm}$ ), the average particle size of AuNP capped by $\alpha$-CD decreased about $40 \mathrm{~nm} .{ }^{41}$ Our results were consistent with this report data that maximum absorption wavelength shifted $1 \mathrm{~nm}$

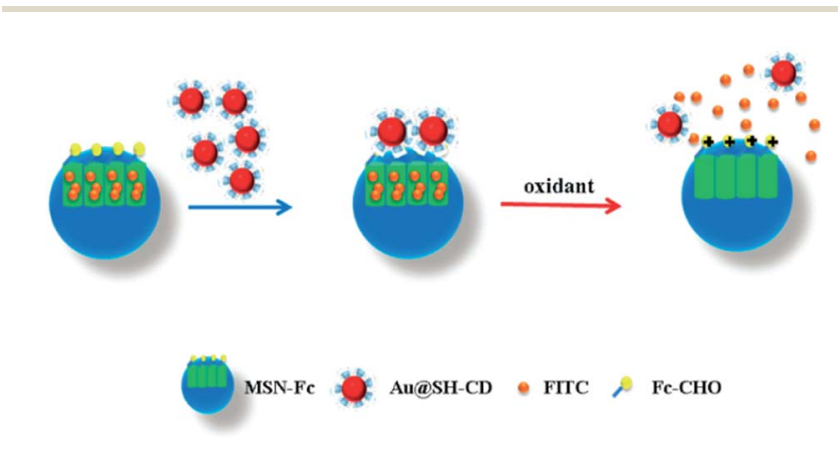

Scheme 1 Schematic diagram of the redox responsive controllable release system based on the functionalized MSN and AuNP.
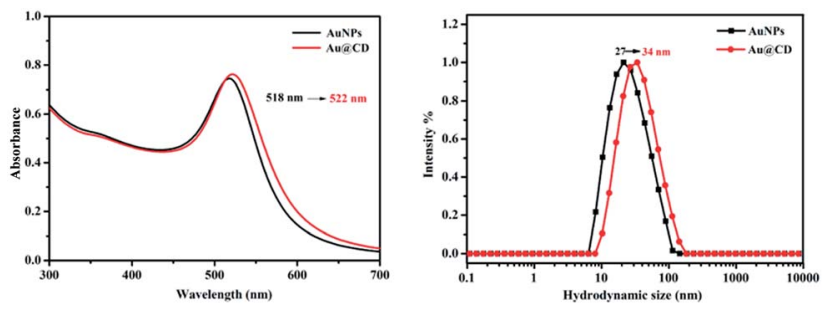

Fig. 1 The UV-visible spectrum (left) and hydrodynamic size distribution (right) of AuNP (black) and Au@CD (red). 
corresponding to average particle size changed 3-4 $\mathrm{nm}$. It was confirmed that the AuNP was successfully functionalized with $\beta$ cyclodextrin. In addition, the AuNP has good dispersion in the water. However, when there was low concentration salt in solution, the aggregation would happen because the negatively charged citrate ions of surface were neutralized, resulting in the color and UV-visible absorption spectrum shifting. Nevertheless, the AuNP@CD showed good dispersion in salt solution, because the modified cyclodextrin can provide steric stabilization. These results confirmed the successful functionalization of AuNP with $\beta$-cyclodextrin.

MSN was prepared in a typical sol-gel process according to the reported literature procedure, and the diameter of MSN was about 100-200 nm. This process involved the hydrolysis and condensation of TEOS in a basic aqueous solution of CTAB template. Then, the amino group was post-grafted onto the surface of MSN to prepare the MSN-NH $\mathrm{N}_{2}$ by surface silylation treatment with APTES. Next Fc was connected to the amino group of surface through shiff-base reaction to obtain the Fc modified MSN (MSN-Fc) (Scheme 2). FT-IR and zeta potential were applied to confirm the modification process. As shown in Fig. 2, the results revealed that the zeta potential for the MSN was $-24.23 \mathrm{mV}$. Due to the introduction of positively charged amino groups after modification, the zeta potential changed to $32.45 \mathrm{mV}$. And the zeta potential of MSN-Fc was $39.23 \mathrm{mV}$. When MSN capped with AuNP@CD, the zeta potential turned back to $-27.15 \mathrm{mV}$ because of the negative charged AuNP. The successfully synthesis of MSN was validated by FT-IR spectrum and there did not exist the stretching vibration of $\mathrm{C}-\mathrm{H}$ at 2950 to $2850 \mathrm{~cm}^{-1}$ from CTAB by removing template through calcine. As can be seen from Fig. 2, the asymmetric vibration of $\mathrm{Si}-\mathrm{OH}$ at $957 \mathrm{~cm}^{-1}$ decreased and a new bending vibration of $\mathrm{N}-\mathrm{H}$ from $\mathrm{NH}_{2}$ group was observed at $1544 \mathrm{~cm}^{-1}$ after attachment of APTES. Fc-CHO has the characteristic peaks at $1680 \mathrm{~cm}^{-1}(\mathrm{C}=\mathrm{O}$ stretching vibration), $1455 \mathrm{~cm}^{-1}$ ( $\mathrm{C}=\mathrm{C}$ stretching vibration), $1100 \mathrm{~cm}^{-1}$ (C-C deformation vibration), $1000 \mathrm{~cm}^{-1}(\mathrm{C}-\mathrm{H}$ deformation vibration), $820 \mathrm{~cm}^{-1}$ (C-H deformation vibration) and $475 \mathrm{~cm}^{-1}$ (C-Fe stretching vibration). However, those characteristic peaks were not obvious because of the overlap with vibration of $\mathrm{Si}-\mathrm{O}-\mathrm{Si}\left(1070 \mathrm{~cm}^{-1}\right)$ and $\mathrm{Si}-\mathrm{O}\left(800 \mathrm{~cm}^{-1}, 460\right.$ $\mathrm{cm}^{-1}$ ). X-ray photoelectron spectroscopy (XPS) was used to further confirm the modification of MSN (Fig. 3). The XPS survey spectrum of the MSN, MSN-NH Fc@Au-CD showed peaks of C1s $(284.6 \mathrm{eV})$, O1s $(532.5 \mathrm{eV})$
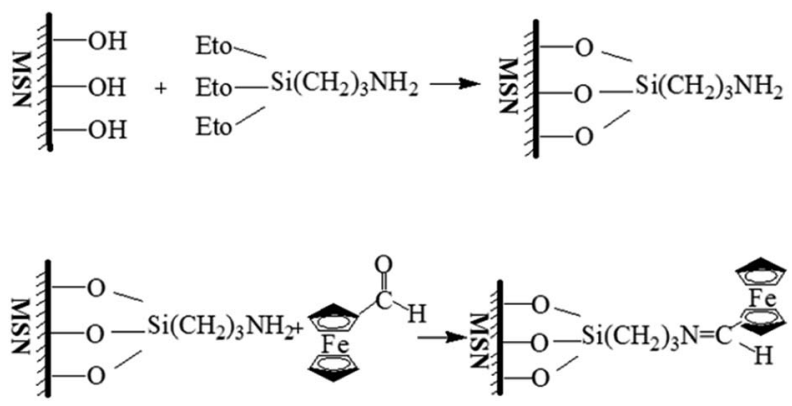

Scheme 2 Synthetic route of the MSN-Fc.
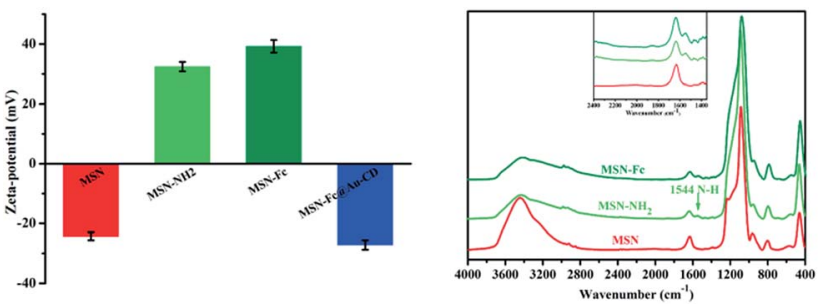

Fig. 2 Zeta potential results (left) and FT-IR spectrum (right) of the $\mathrm{MSN}$ (red), MSN-NH (green), MSN-Fc (olive green) and MSN-Fc@AuCD (blue).

and Si2p (103.18 eV) in Fig. 3A. As can be seen from Fig. 3B, the high-resolution XPS N1s spectrum of $\mathrm{MSN}-\mathrm{NH}_{2}$ indicated the presence of amino group. In addition, the presence of the $\mathrm{Fc}$ is confirmed from the high-resolution Fe2p spectrum in Fig. 3C and this result indicated that Fc has been modified on the MSN successfully. The Au4f peaks at the binding energies of 84.4 and $88.05 \mathrm{eV}$, as shown in Fig. 3D, are attributed to $\mathrm{Au} 4 \mathrm{f}_{7 / 2}$ and Au4f $_{5 / 2}$, respectively. The two strong Au characteristic peaks suggest clearly the binding of AuNP with good crystallization. These results confirmed that the modification of MSN was successful. This is the key factor for the controlled release system to change the strength of host-guest interactions by oxidation of the Fc.

Moreover, the porous properties of the MSN and functionalized MSN were measured by BET nitrogen adsorptiondesorption isotherms and $\mathrm{BJH}$ pore size distribution analysis, as was shown in Fig. 4. The BET isotherm of MSN exhibited the characteristic type of IV $\mathrm{N}_{2}$ adsorption-desorption patterns according to the IUPAC classification, which indicated that the particles possessed uniform mesoporous channels. The BET surface of the MSN, MSN-Fc and MSN-Fc@Au-CD were $608.7 \mathrm{~m}^{2}$ $\mathrm{g}^{-1}, 96.4 \mathrm{~m}^{2} \mathrm{~g}^{-1}$ and $226.3 \mathrm{~m}^{2} \mathrm{~g}^{-1}$, respectively (Table 1 ). The changes in the adsorption isotherm and the surface area of the

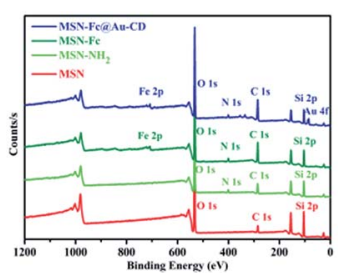

A

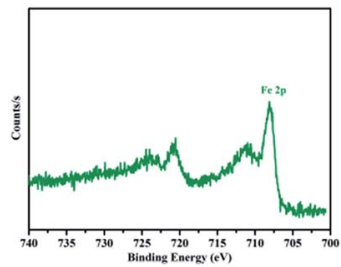

$\mathrm{C}$

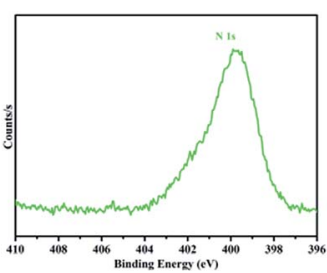

B

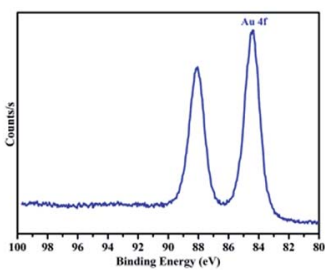

$\mathrm{D}$
Fig. 3 XPS spectra of (A) MSN (red), MSN-NH (green), MSN-Fc (olive green) and MSN-Fc(aAu-CD (blue). High resolution (B) N1s, (C) Fe2p and (D) Au4f spectrum of MSN-NH $\mathrm{N}_{2}, \mathrm{MSN}-\mathrm{Fc}$ and MSN-Fc@Au-CD, respectively. 


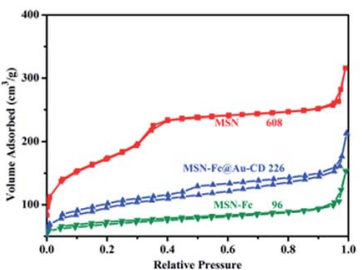

A

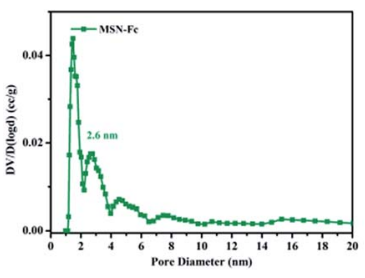

$\mathrm{C}$

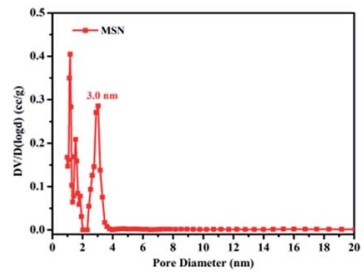

B

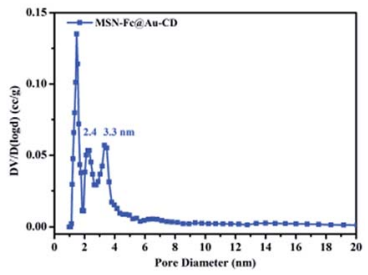

$\mathrm{D}$

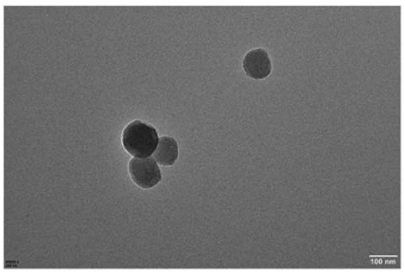

A

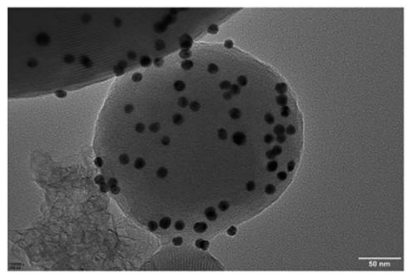

C

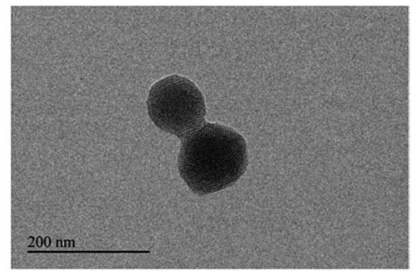

B

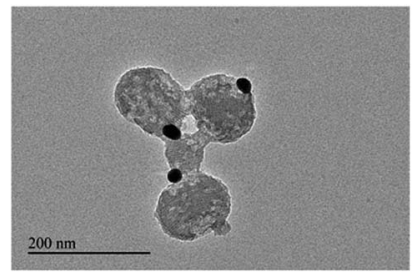

D
Fig. 4 Nitrogen adsorption-desorption isotherms (A) and BJH pore size distribution (B-D) for MSN (red), MSN-Fc (olive green) and MSNFc@Au-CD (blue).

Table 1 BET and BJH parameters

\begin{tabular}{lcl}
\hline Samples & $S_{\text {BET }}\left(\mathrm{m}^{2} \mathrm{~g}^{-1}\right)$ & Pore volume $\left(\mathrm{cm}^{3} \mathrm{~g}^{-1}\right)$ \\
\hline MSN & 608.7 & 0.488 \\
MSN-Fc & 96.4 & 0.206 \\
MSN-Fc@Au-CD & 226.3 & 0.325
\end{tabular}

FITC-loaded and AuNP@CD capped MSN-Fc were due to the FITC loading and AuNP@CD capping. Meanwhile, the pore size of MSN-Fc was decreased compared with MSN because of Fc modification (Fig. 4).

The morphology of the MSN and functionalized MSN-Fc has been studied by transmission electron microscopy (TEM). Fig. 5 revealed that MSN were spherical nanoparticles with hexagonally arranged pores. The shape and size of the particles were retained after modification with Fc. The pore structure of the FITC-loaded and AuNP@CD capped MSN-Fc cannot be observed clearly after cargo loading and AuNP@CD capping because of the FITC incorporation in the pores and the AuNP@CD capping on the opening of the pores lead to a lower image contrast between the channel and the silica wall. In conclusion, we successfully developed nanocarriers using the Fc functionalized MSN and the gatekeeper AuNP@CD.

\section{Redox responsive controlled release of FITC}

In order to investigate the redox responsive controlled release behavior of this self-assemble system, the excess FITC molecules were added to the MSN-Fc solution, and enable them to fully fill the pores of the MSN-Fc. After that, the MSN-Fc@Au-CD was centrifuged and washed with PBS buffer, then was dried under $60{ }^{\circ} \mathrm{C}$ in vacuum. The loading of the FITC was determined approximately $383.98 \mathrm{nmol} \mathrm{mg}^{-1}$ according to the characteristic absorption peak at $490 \mathrm{~nm}$. TEM image revealed that the FITC
Fig. 5 TEM image of MSNs. (A) MSN (B) MSN-FC-FITC (C) MSNFc@Au-CD (D) MSN-Fc@Au-CD addition with $\mathrm{H}_{2} \mathrm{O}_{2}$.

was loaded in the pores of MSN. Moreover, the changes of pore volume and diameter also can further confirm the FITC loading.

This redox responsive controllable release system is based on the interaction of ferrocene and $\beta$-CD. When AuNP@CD interacts with ferrocene group which have been modified on the pore orifices of the MSN, the inclusion complexes become caps to prevent FITC release. Normally, uncharged Fc species or their derivatives are strongly bonded in the hydrophobic cavities of $\beta$ $\mathrm{CD}$, whereas the charged species $\left(\mathrm{Fc}^{+}\right)$dissociate rapidly from the cavities.

The method we used to oxidate $\mathrm{Fc}$ to $\mathrm{Fc}^{+}$is using $\mathrm{H}_{2} \mathrm{O}_{2}$ as oxidant agent. As shown in Fig. 6, the guest molecules FITC were released from the pores with the addition of $\mathrm{H}_{2} \mathrm{O}_{2}$. The peak of FITC at $515 \mathrm{~nm}$ was significant after released for $120 \mathrm{~h}$ with addition of $0.01 \% \mathrm{H}_{2} \mathrm{O}_{2}$ in this fluorescence spectrum. Compared with reported nanoparticles capped MSN,$^{14} \mathrm{MSN}-$ Fc@CD didn't showed a "zero premature release" due to the uncompletely cover. However, the AuNP@CD as gatekeeper can still have a better sealing effect compared with bare $\beta$-CD in the same conditions (Fig. 6). The adsorption and desorption of AuNP@CD from the surface of MSN-Fc was also characterized by TEM (Fig. 5). The TEM images demonstrated that the AuNP@CD was almost removed to the solution with the addition of $\mathrm{H}_{2} \mathrm{O}_{2}$. Subsequently, the redox responsive release behavior of FITC with different amounts of $\mathrm{H}_{2} \mathrm{O}_{2}$ addition was
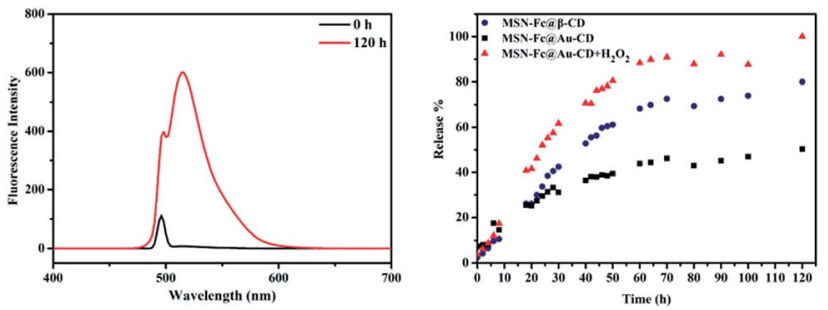

Fig. 6 The fluorescence spectrum of released FITC (left) and release behavior after addition of $\mathrm{H}_{2} \mathrm{O}_{2}$ (right). 


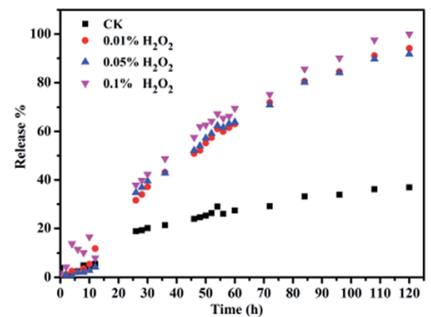

Fig. 7 The release behavior of FITC over time with the addition different amount of $\mathrm{H}_{2} \mathrm{O}_{2}$.

also investigated using fluorescence spectrum as shown in Fig. 7. FITC was released slowly and there was no significant difference with different amounts of $\mathrm{H}_{2} \mathrm{O}_{2}$ addition. The fluorescence intensity of supernatant was low without stimuli, which also indicated that the AuNP@CD had good capping efficiency. Xiao et al. developed an enzyme and voltage responsive release system based on $\beta$-CD capped MSN which can release more than $80 \%$ loading molecular in $18 \mathrm{~h}$ under stimulus. ${ }^{8}$ Another enzyme controlled drug delivery system based on polyglutamin acid-gated MSN can release $90 \%$ of the entrapped guest in $5 \mathrm{~h}$ in the presence of pronase. ${ }^{42}$ The release time in our work is much longer than those responsive systems which mainly within $24 \mathrm{~h}$. There are many factors can affect either the loading amount of the guest molecular or the release behavior such as pore size, pore structure, surface functionalization. ${ }^{43,44}$ For example, MCM-41 loaded with ibuprofen with $2.5 \mathrm{~nm}$ diameter can total release the drug in $48 \mathrm{~h}$, while the MCM-41 functionalized with aminopropyl with $1.7 \mathrm{~nm}$ pore diameter need $213 \mathrm{~h}$ to finish this release behaviour. ${ }^{45}$ It has been found that the release becomes slower with the pore size of the matrix decreasing or the molecular size of the loading molecule increasing. ${ }^{46}$ The pore diameter of MSN and MSN-Fc is more than $2.6 \mathrm{~nm}$ according to the $\mathrm{BJH}$ pore size distribution analysis, however, they also both exist $1.5 \mathrm{~nm}$ pore diameter (Fig. 4). As the literature revealed, MSN with smaller pore diameter would lead to a slower release behavior. Another possibility is that FITC has some aromatic rings in the structure which may interact with $\mathrm{Cp}$ ring of ferrocene and will block the release behavior. Due to this slow release behavior, this kind of redox responsive controllable release system can be used as a sustained drug delivery system.

\section{Conclusions}

In this work, we successfully developed a redox responsive controlled release system through the host-guest interactions between $\beta$-CD modification on AuNP (Au@CD) and Fc modification on the surface of MSN (MSN-Fc). The ferrocene functionalized MSN can be used as the host materials for small molecular loading and the $\beta$-cyclodextrin functionalized AuNP can be used as the gate-controlled materials. And FITC was selected as the release molecule. The Fc molecule showed different interactions with $\beta$-cyclodextrin under different oxidations. Uncharged ferrocene or their derivatives are strongly bonded into the hydrophobic cavities of $\beta$-CD, whereas the charged species dissociate rapidly from the cavities. This controlled release system can be used as targeted delivery drugs and the release behavior can be realized through addition of oxidizing agents such as $\mathrm{H}_{2} \mathrm{O}_{2}$. This new type of redox responsive controllable sustained release system will have a potential application in vivo due to the good biocompatibility and easy to image detection of AuNP.

\section{Acknowledgements}

This work was supported by the National Natural Science Foundation of China (21676273), National Key Natural Science Foundation of China (U1507203), the National Technology Research and Development Program of China (2015CB251402), the Youth Innovation Promotion Association, CAS (Grant No. 2016043).

\section{References}

1 M. Vallet-Regi, A. Rámila, R. P. D. Real and J. Pérez-Pariente, Chem. Mater., 2000, 13, 308-311.

2 C. Argyo, V. Weiss, C. Bräuchle and T. Bein, Chem. Mater., 2013, 26, 435-451.

3 Y. Chen, H. Zhang, X. Cai, J. Ji, S. He and G. Zhai, RSC Adv., 2016, 6, 35757-35761.

4 Z. S. Alahmady, W. T. Aljamal, J. V. Bossche, T. T. Bui, A. F. Drake, A. J. Mason and K. Kostarelos, ACS Nano, 2012, 6, 9335-9346.

5 H. Meng, M. Xue, T. Xia, Y. L. Zhao, F. Tamanoi, J. F. Stoddart, J. I. Zink and A. E. Nel, J. Am. Chem. Soc., 2010, 132, 12690-12697.

6 D. Wang and S. Wu, Langmuir, 2015, 32, 632-636.

7 S. Mura, J. Nicolas and P. Couvreur, Nat. Mater., 2013, 12, 991-1003.

8 Y. Xiao, T. Wang, Y. Cao, X. Wang, Y. Zhang, Y. Liu and Q. Huo, Dalton Trans., 2015, 44, 4355-4361.

9 X. Chen, X. Cheng, A. Soeriyadi, S. Sagnella, X. Lu, J. Scott, S. Lowe, M. Kavallaris and J. J. Gooding, Biomater. Sci., 2013, 2, 121-130.

$10 \mathrm{H}$. He, H. Kuang, L. Yan, F. Meng, Z. Xie, X. Jing and Y. Huang, Phys. Chem. Chem. Phys., 2013, 15, 14210-14218.

11 S. Kim, S. Philippot, S. Fontanay, R. E. Duval, E. Lamouroux, N. Canilho and A. Pasc, RSC Adv., 2015, 5, 90550-90558.

12 P. D. Ray, B.-W. Huang and Y. Tsuji, Cell. Signalling, 2012, 24, 981-990.

13 T. C. Jorgenson, W. Zhong and T. D. Oberley, Cancer Res., 2013, 73, 6118-6123.

14 S. Giri, B. G. Trewyn, M. P. Stellmaker and V. S. Lin, Angew. Chem., 2005, 44, 5038-5044.

15 E. Aznar, M. Oroval, L. Pascual, J. R. Murguía, R. Martínezmáñez and F. Sancenón, Chem. Rev., 2016, 116, 561.

16 E. Aznar, M. D. Marcos, R. Martínez-Máñez, F. Sancenón, J. Soto, P. Amorós and C. Guillem, J. Am. Chem. Soc., 2009, 131, 6833. 
17 R. Liu, X. Zhao, T. Wu and P. Feng, J. Am. Chem. Soc., 2008, 130, 14418-14419.

18 Y. Cui, H. Dong, X. Cai, D. Wang and Y. Li, ACS Appl. Mater. Interfaces, 2012, 4, 3177-3183.

19 L. Chen, Z. Zheng, J. Wang and X. Wang, Microporous Mesoporous Mater., 2014, 185, 7-15.

20 Q. Yan, J. Yuan, Z. Cai, Y. Xin, Y. Kang and Y. Yin, J. Am. Chem. Soc., 2010, 132, 9268-9270.

21 D. Qunpeng, C. Yu, L. Yan, H. Xiaoyu, X. Tangxin, L. Chen, P. Yi and W. Leyong, J. Am. Chem. Soc., 2013, 135, 1054210549.

22 Y. L. Sun, Y. W. Yang, D. X. Chen, G. Wang, Y. Zhou, C. Y. Wang and J. F. Stoddart, Small, 2013, 9, 3224-3229.

23 H. Xuan and X. Du, ACS Appl. Mater. Interfaces, 2014, 6, 20430.

24 K. C. Leung, T. D. Nguyen and J. F. Stoddart, Chem. Mater., 2006, 18, 5919-5928.

25 S. Angelos and K. Patel, Angew. Chem., 2008, 47, 2222.

26 S. Angelos, N. M. Khashab, Y. W. Yang, A. Trabolsi, H. A. Khatib, J. F. Stoddart and J. I. Zink, J. Am. Chem. Soc., 2009, 131, 12912-12914.

27 Y. L. Sun, Y. Zhou, Q. L. Li and Y. W. Yang, Chem. Commun., 2013, 49, 9033.

28 Y. L. Zhao, Z. Li, S. Kabehie, Y. Y. Botros, J. F. Stoddart and J. I. Zink, J. Am. Chem. Soc., 2010, 132, 13016-13025.

29 M. Nakahata, Y. Takashima, H. Yamaguchi and A. Harada, Nat. Commun., 2011, 2, 511.

30 H. Yang, B. Yuan, X. Zhang and O. A. Scherman, Acc. Chem. Res., 2014, 47, 2106-2115.

31 A. J. Evans, S. E. Matthews, A. R. Cowley and P. D. Beer, Dalton Trans., 2003, 4644-4650.
32 L. Peng, A. Feng, M. Huo and J. Yuan, Chem. Commun., 2014, 50, 13005-13014.

33 A. Harada and S. Takahashi, J. Chem. Soc., 1984, 10, 645-646. 34 A. Hashidzume, H. Yamaguchi and A. Harada, in Molecular Machines and Motors, Springer, 2014, pp. 71-110.

35 X. L. Qiu, Q. L. Li, Y. Zhou, X. Y. Jin, A. D. Qi and Y. W. Yang, Chem. Commun., 2015, 51, 4237-4240.

36 L. Tan, H. X. Wu, M. Y. Yang, C. J. Liu and R. X. Zhuo, RSC Adv., 2015, 5, 10393-10399.

37 D. P. Ferris, Y. L. Zhao, N. M. Khashab, H. A. Khatib, J. F. Stoddart and J. I. Zink, J. Am. Chem. Soc., 2009, 131, 1686-1688.

38 C. Chen, F. Pu, Z. Huang, Z. Liu, J. Ren and X. Qu, Nucleic Acids Res., 2011, 39, 1638-1644.

39 M. Oroval, E. Climent, C. Coll, R. Eritja, A. Aviñó, M. D. Marcos, F. Sancenón, R. Martínezmáñez and P. Amorós, Chem. Commun., 2013, 49, 5480-5482.

40 J. Liu and Y. Lu, Nat. Protoc., 2005, 1, 246-252.

41 T. Huang, F. Meng and L. Qi, J. Phys. Chem. C, 2009, 113, 13636-13642.

42 A. Tukappa, A. Ultimo, L. T. C. De, T. Pardo, F. Sancenón and R. Martinezmañez, Langmuir, 2016, 32, 8507-8515.

43 S. K. Natarajan and S. Selvaraj, RSC Adv., 2014, 4, 1432814334.

44 M. Vallet-Regí, Chem.-Eur. J., 2006, 12, 5934-5943.

45 A. Rámila, B. Muñoz, J. Pérez-Pariente and M. Vallet-Regí, J. Sol-Gel Sci. Technol., 2003, 26, 1199-1202.

46 I. Izquierdo-Barba, L. Ruiz-González, J. C. Doadrio, J. M. González-Calbet and M. Vallet-Regí, Solid State Sci., 2005, 7, 983-989. 